\title{
ÉLECTRICITÉ
}

\section{Un nouvel appareil de prise de vues ultra-rapide pour projections cinématographiques à l'extrême ralenti}

par M. Gérardin, Ingénieur I. E. G. et E. S.E.

\section{INTRODUCTION}

La cinématographie est, à lheure actuelle, arrivée à un tel degré de perfectionnement technique, qu'il semble, au premier abord, présomptueux de chercher à réaliser la prise de vue suivant un principe différent de celui consacré par l'usage. On peut bien dire, en effet, que, dès son origine, les efforls de tous les chercheurs ont eu pour unique bul de perfectionner le principe initial consistant à dérouler le film de façon intermittente, par saccades, et à l'impressionner pendant ses périodes d'immobilité, au moyen d'un obturateur périodique judicieusement synchronisé. Tant qu'il s'agit de reproduire un phênomène à sa vitesse réelle cette méthode d'enregistrement est parfaite ; mais sit l'on cherche ò faire une analyse beaucoup plus détaillée du mouvement, de façon à permettre la projection du phénomène au ralenti, on se heurle, à nouveau, à des difficulíés mécaniques tenanl à la délicatesse des mécanismes d'entrẩnement du film, et à la fragilité de celui-ct. Il faut penser, en effet, que la prise de 5.000 vues à la seconde par exemple sur le film normal $19 \times 24 \mathrm{~m} / \mathrm{m}$, exigerait une vitesse moyenne de $5 \times 19$, soil cnviron $95 \mathrm{~m}$. à la seconde et en tenant compte de l'arrêt sur chaque vue, d'une vilesse moyenne intermitlente de $190 \mathrm{~m}$. ce qui correspondrait à une accélération de $7.600 \mathrm{Km} . / \mathrm{sl}$. On comprend aisément par ces chiffres, que malgré lo jaible masse du film, sa résistance serait inférieure aux forces d'inertie qu'il aurait à supporter ; de même d'ailleurs pour les organes de transmissior du mouvement. C'est pourquoi celte technique courante ne permet guère de prendre plus de deux cents vues par seconde, ce qui, compte tenu du fait qu'une projection normale utilise environ 16 images par seconde, permet d'oblenir sur l'écran un ralenti de 1/12 environ. On concrétisera aisément ce chiffre si l'on songe que c'est le ralenti couramment employé lorsqu'il sagit, par exemple, de décomposer les mouvements a'un athlète au cour's d'une performance.

Ce n'esit là, évidemment, encore, qu'une timide incursion dans le domaine de linfiniment conrt, et, pour quelque intéressante qu'elle soit, cette investigalion nous permet seulement de mieux connaitre certaines classes de phénomènes, dont l'évolution est déjà accessible à nos sens. ; mais elle est incapable de nous offrir la révétation d'un monde nouveau. Or, il existe une foule de phénomènes dont le processus nous échappe presque complètement; seuls, quelques instantanés photographiques nous permettent de nous en faire une idée, forcémenl à priori ; el, pour n'en énumérer gne quelques-uns on peut citer ceux ayant trait aux ares ćlectriques - certains phénomènes d'ordre biologique tels que le vol des insectes - une quantité de phénomènes, tels que vibrations, chocs, cassures - des phétumines acoustiques. - les decharges bectriques, etc... Il convenail done pour les chercheurs de s'orienler vers d'autres méthodes de prise de vaes utitisant, en paritalier, an deronlement contimu du film.

\section{IIISTOMLED}

\section{Premières tentatives d'utilisation d'un déroulement continu du film pour la prise de vues rapide.}

Il est curieux de constater que les précurseurs du cinéma, el, entre autres, tes biologistes de l'Ecole de Marey, s'étaient déjà intéressés à l'ultra ralenti, notamment en ce qui concerne le vol des insectes, à une époque où il n'ólait encore nullement question de cinématographie à proprement parler.

Leur dispositif expérimental consistait en un tambour $\mathrm{T}$, tournant à une vitesse angulaire uniforme autour d'un axe penpendieulatre an jlan de la figure, une pellicule photographique était enroulée autour de ce tambour, et devant celui-ci se trouvait un objectif muni d'un diaphragme. L'objectif élait placé de façon à donner du phénomène à étudier, en l'espèce un insecte enformé dans une cage de verre, whe image nette sur la pellicule photographique.
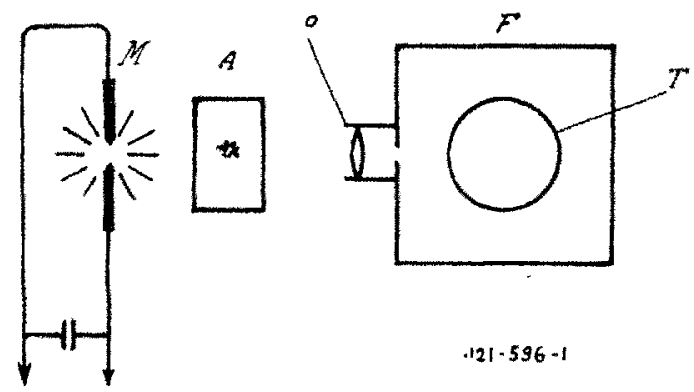

FIG.

L'éclaircment du sujet était réalisé en utilisant la décharge d'une batterie de condensateurs, it travers deus électrodes de magnésium. Cette décharge durail un temps extrêmement court, de l'ordre de $10^{-5}$ seconde. Un système de contacts tournants, non représentć sur la figure, permettail de la répéter 2.000 fois par seconde. La vitesse du liımbour ćlait réglée de façon que le film se dóplace de la hauteur d'une image, dans l'intervalle de temps compris entre deux éclairs : c'est-à-dire tous les $1 / 2000$ de seconde. On voit aussi que pendant le temps d'exposition de l'image (10-5 seconde), celle-ci ne s'est déplacée par rapport au film que de $\frac{10-5}{1 / 2.000}$ sod $\frac{1}{50}$ de sa hauteur, déplacement encort 
admissible à la rigueur polur sauvegarder une certaine netteté des images.

Un obturateur spécial permettail de n'impressionner le film que pendint un tour de tambour.

Ce dispositif, de par sa nature mème, présentait le défaut capital de ne pouvoir servir à l'étude des phónomènes lumineux tels que les arcs électriques de fréquence industrielle. Néanmoins, malgré ses imperıeclions, ce dispositif présente la particularité très intéressante d'utiliser un déroulement continu du film à vitesse uniforme, et c'est dans cette voie que s'engagèrent, par la suite, tous ceux qui s'intéressèrent du problème de l'ultra-ralenti.

\section{Situation actuelle de la technique des appareils de prise de vues rapide}

Il semble que jusqu'ici, lidée dominante des chercheurs, ait été d'obtenir le plus gnand nombre possible d'images pendant le minimum de temps. Au premier abord, cela parait bien naturel, et l'on pourritil s'étonner qu'il en fùt autrement. Néanmoins, ce n'est pas, à notre avis, le côté le plus intéressant de la question. C'est évidemmenl une chose que de prendre le maximum de rues pendant le minimum de temps, sans restrictions d'aucune sorte ; mais, c'en est une autre que de s'imposer, en outre, l'obligation de les projeter ensuite au ralenti sur un écran. sans l'intermédiaire d'opérations délicates de reproduction ou d'appareils spéciaux de projection.

Si l'on s'en tient à la première formule, on n'aura jamais qu'une sẹ́rie d'images inanimées, en quelque sorte, un phénomène en pièces détachées, dont on ne peut rcconstituer le processus que par l'imagination.

La seconde nous permet, au contraire, de reconstituer réellement et visuellement le phénomène, dans son essence mème qui est le temps : l'échelle seule est changée pour l'adapter aux imperfections de l'organisme humain.

Si nous insistons 'un peu sur ce point, c'est que justement, les procédés actuellement en usage pour obtenir le plus grand nombre possible de vues pendant le minimum de lemps, s'opposent par leur nature même à la projection cinématographique.

Les appareils réalisés sont des appareils photographiques et non point des appareils cinématographiques. Ils ont, en effet, tous pour principe, la réalisation sur le film d'une série d'images, non plus disposées seulement les unes à la suite des autres, dans le sens du déplacement du film (fig. 2), mais encore juxtaposées en mème temps, par séries de 4 ou 5 vues successives, lés unes à côté des autres dans le sens
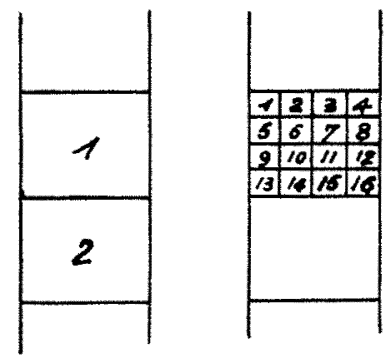

$$
\text { Fig. } 2 \text { Fig. } 3
$$

transversal (fig. 3). Ces images se succèdent donic à la façon des leltres dans un textc (dans les lig. 2 el 3 ces images sont numérotées suivant leur ordre de succession dans le temps). Si l'on fait abstraction de l'impossibilité de pro- jeter un lel assemblage de vues, on voit tout de suite l'avanlage de cc procédé qui, pour une même vitesse de déroulement du film, permet dans le cas de la fig. 3 d'obtenir 16 fois plus d'images pendant le même temps, mais de dimensions réduites, évidemment, dans la même proportion.

\section{I. - Appareils de prise de vues à images fixes}

Deux modes opératoires en présence, tous deux utilisant, comme nous l'avons déjà signalé, un déroulement du film ì vitesse uniforme.

Le premier, spécialement étudié et mis au point par le professeur Nagnan, au cours de ses études sur le vol des insectes, peut se décrire de la façon suivante :

Un objectif donne du phénomène à étudier, une image nette sur le film ; mais, celui-ci se déroulant à une vitesse uniforme el l'image étant immobile, on esst obligé, d'une part, dimpressionner le film pendant un temps suffisamment court, pour que le déplacement relatif $\varepsilon$ de l'image, par rapport au film, soit petit en comparaison de la hau-

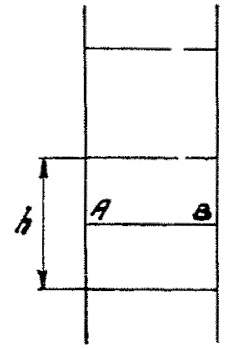

Fig. 4
Fig. 5

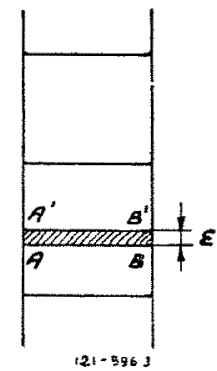

teur $h$ de l'image (roir fig. 4 et 5 ), et d'autre part, de répéter cetle opération chaque fois que le film s'est déplacé d'une distance égale à la hauteur des images.

Ceci est réalisé très simplement au moyen d'un disque tournant, sur la périphérie duquel sont ménagées des fentes

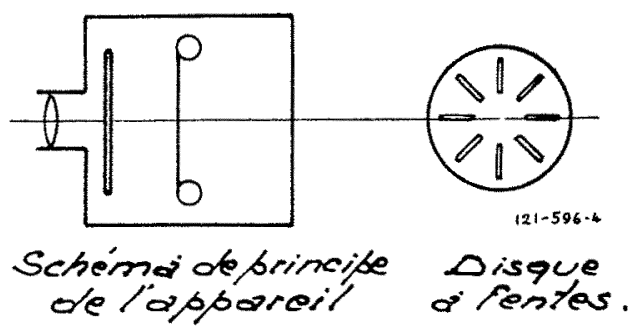

Fig. 6

radiales, régulièrement et judicieusement espacées défilant tour à tour derrière l'objectif, au niveau de l'axe optique de celui-ci (voir fig. 6). Ce dispositif, qui réalise en quelque sorte un oblurateur de plaque, a l'avantage de la simplicité et de l'économie. De plus, la partie optique se réduisant à un seul objectif, ce dernier peut être de toute première qualité.

Malheureusement, pour bénéficier au maximum de ses qualités optiques, il faudrait que pendant le temps d'exposition le déplacement du film soit aussi faible que possible, d'où pour une vitesse donnée du film, la nécessité d'avoir des fentes d'une très grande finesse : la luminosité de l'appareil est par suite très faible. En fait, on ne peut guère songer à éludier avec ce dispositif que des phénomènes éclairés à contre-jour, c'est-à-dire des silhouettes.

Le second, imaginé par les biologistes de l'école de Marey, 
el perfectionné réceriment par la société stroborama, consiste ainsi que nons lavons déjà relité, à éclairer le sujet à cinématographier, par intermittences. La durée de l'éclairement étant petite par rapport an temps que met le film à se déplacer de la hauteur d'une image, nous avons déjà signalé les avantagés ct les inconvénients de ce système.

\section{II. - Appareils de prise de vues à images mobiles}

Puisque le film est animé d'une vitesse de déroulement uniforme, il vient tout naturellement à l'esprit d'essayer de communiquer à l'image une vitesse égale et de même sens que celle du film, au moment de l'impression, de façon à rendre l'image fixe par rapport au film. On pourra de la sorte, impressionner le film pendant tout le temps qu'il met à se déplacer de la hauteur d'une image, et réaliser, par' suite, un gain considérable de luminosité comparativement aux dispositifs à images fixes.

\section{Premier procédé de réalisation de la fixité des images par rapport au film.}

Comment obtenir cette fixité ?

Un premier procédé consiste à monter à la périphérie d'un disque tournant, une série d'objectifs équidistants. Ce dis-

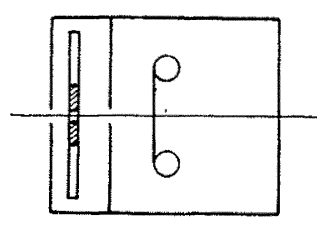

Fig. 7

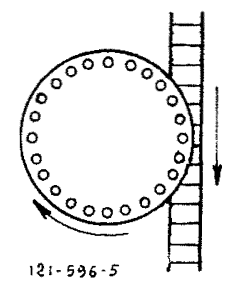

$$
121.536-5
$$

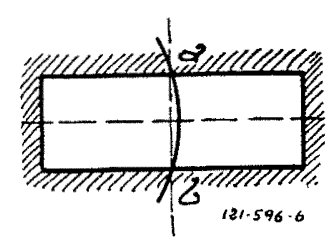

Fig. 8 que est disposé de façon telle que les objectifs viennent, tour à tour, défiler au niveau d'une ouverture derrière laquelle se déplace le film (figure 7), la hauteur de l'ouverture étant suffisamment faible pour que l'arc de cercle décrit par le centre optique d'un objectif au cours de son trajet devant l'ouverture puisse être confondu avec un segment de ḋroite (fig'ure 8).

La condition à réaliser est alors que lid vitesse tangentielle des lentilles au niveau de l'ouveriure snit égale à la vilesse d. déplacement du film. En effet. considérons un objectif de centre $O$, de foyer $F$ et d'axe optique $\Delta$ (iig. (3). Un point $\mathrm{P}$ situé à l'infini sur l'axe $\Delta$ viendra former son image en $\mathrm{F}$.

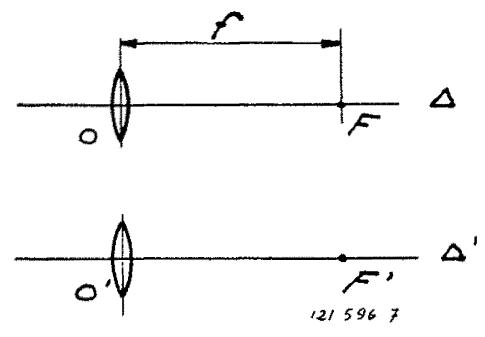

Fig. 9

Si cet objectif se déplace parallèlement à lui-même en $\mathrm{O}^{\prime}$ $\Delta$ vient en $\Delta^{\prime}$, le point $\mathrm{P}$,viendra former son image en $\mathrm{F}^{\prime}$, le déplacement FF étaut égal au déplacement $O \mathrm{O}^{\prime}$ et ayant lieu pendant le même temps. On voit que la vitesse de l'image donnée par l'objectif $O$ est égale à la vilesse de cet objectif. Il suffira que la vitesse du film soit égale à celle de l'objectif pour réaliser l'immobilité de l'image par rapport au film.

INGONVÉNIENTS DE GE PROCF́dÉ.

a) L'immobililé de l'image par rupport al film n'esl jamais parfaitement réalisée.

L'immobilité de l'image par rapport au film cst véalisée tant que le point $P$ est très éloigné, c'est-à-dire, tant que la

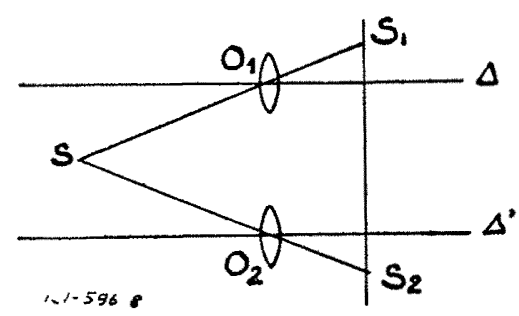

Fig. 10

dislance OP est grande par rapport ì OF, mais ne l'est plus lorsque ces distances sont du même ordre de grandeur. En effet, un point tel que $\mathrm{S}$ (fig. 10) donnera une image $\mathrm{S}_{\mathrm{I}}$ lorsque l'objectif est en $\mathrm{O}_{1}$, une image $\mathrm{S}_{2}$ lorsqu'il est en $O_{2}$ et il est évident que $\mathrm{S}_{1} \mathrm{~S}_{2}<\mathrm{O}_{1}\left(\mathrm{O}_{2} ; \mathrm{S}_{1} \mathrm{~S}_{2}\right.$ étant d'autant plus grand que le point $\mathrm{S}$ est plus rapproché de l'objectif, d'où un premier et grave inconvénient du procédé.

b) Difficultés d'obtenir à ln fois un très grand nombre d'images sur le film et une distance convenable entre les objectifs montés sur le disque.

Quelles sont les conditions auxquelles doivent satisfaire les images recueillies sur le film destiné à être projeté à l'aide d'un appareil courant de projection cinématographique.

$1^{\circ}$ ) Ces images doivent être équidistantes, chose facile à réaliser.

$2^{\circ}$ ) Elles doivent de plus se succéder à des intervalles rigoureusement calibrés, variables suivant le type de film courant employé et qui sont de $3,8 \mathrm{~m} / \mathrm{m}$ pour le film de $8 \mathrm{~m} / \mathrm{m}$ (film minimum du commerce) $7 \mathrm{~m} / \mathrm{m} 6$ pour celui de $16 \mathrm{~m} / \mathrm{m}$ et $19 \mathrm{~m} / \mathrm{m}$ pour celui de $35 \mathrm{~m} / \mathrm{m}$ (film normal).

Or, l'expérience montre que; quelles que soient les dimensions du film employé, on ne peut guère dépasser une vitesse de déroulement supérieure à $20-25 \mathrm{~m}$. par seconde, sans risquer de détériorer le film (cisaillement au niveau des encoches). D'autre part, les objectifs montés sur le disque tournant ne peuvent guère avoir un diamètre inférieur à $8 \mathrm{~m} / \mathrm{m}$, si l'on veut réaliser une bonne luminosité $(\mathrm{f} / 3,5)$ et une construction soignée (anastigmats). La situation paraît sans issue, car si l'on veut réaliser un ralenti très poussé, on est fatalement conduit à 'uliliser le film de $8 \mathrm{~m} / \mathrm{m}$ qui est à l'heure actuelle le plus petit film du commerce ; mais les objectifs devront, dans ce cas, être disposés à $3,8 \mathrm{~m} / \mathrm{m}$ de distance les uns des autres, sur la périphérie du disque $(3,8 \mathrm{~m} / \mathrm{m}$ élant le pas du film de $8 \mathrm{~m} / \mathrm{m})$, ce qui est impossible à réaliser pratiquement.

$\mathrm{Si}$ au contraire on donne aux objectifs des dimensions convenables, on perd de ce fait tous les avantages d'un déroulement rapide, étant donné les dimensions importantes des images auxquelles on arrive. 


\section{Second procédé de réalisation de la fixité des images par rapport au film.}

A) Positron du probième.

La considération des imperfections du système précédent nous permel de poser le problème de la façon suivante :

a) Obtenir sur le film des images immobiles par rapport à ce dernier, quelque soit la distance de l'objet à cinémalographier.

b) Oblenir une vitesse de déroulement du film inférieure à la vilesse tangentielle des objectifs mobiles.

On pourrait ainsi :

$\left.1^{\circ}\right)$ Utiliser le plus petit film utilisé dans le commerce, en l'espèce le film de $8 \mathrm{~m} / \mathrm{m}$ dont l'entre-axe des images est de $3,8 \mathrm{~m} / \mathrm{m}$.

$\left.2^{\circ}\right)$ Monter à la périphérie du disque tournant, des objectifs pratiquement réalisables dans de bonnes conditions (objectifs de diamètre supérieur à $8 \mathrm{~m} / \mathrm{m}$ ).

B) SOLUTION DU PROBLÈME PAR L'EMPLOI D'UN OBJECTIF pLaCÉ EN AVANT DU DISQLE TOURNaNT.

Un objectif fixe $M$, mais de tirage réglable, placé en avant du disque tournant et dont l'axe optique cöncide avec celui des objectifs mobiles du disque, lorsqu'ils passent au niveau de l'ouverture 0 , permet la solution complète du problème énoncé ci-dessus.

$\left.1^{\circ}\right)$ Il réalise la fixité parfaite des images par rapport ait film.

En effet, cet objectif étant de tirage réglable peut toujours donner, d'un objet placé à une distance quellconque,

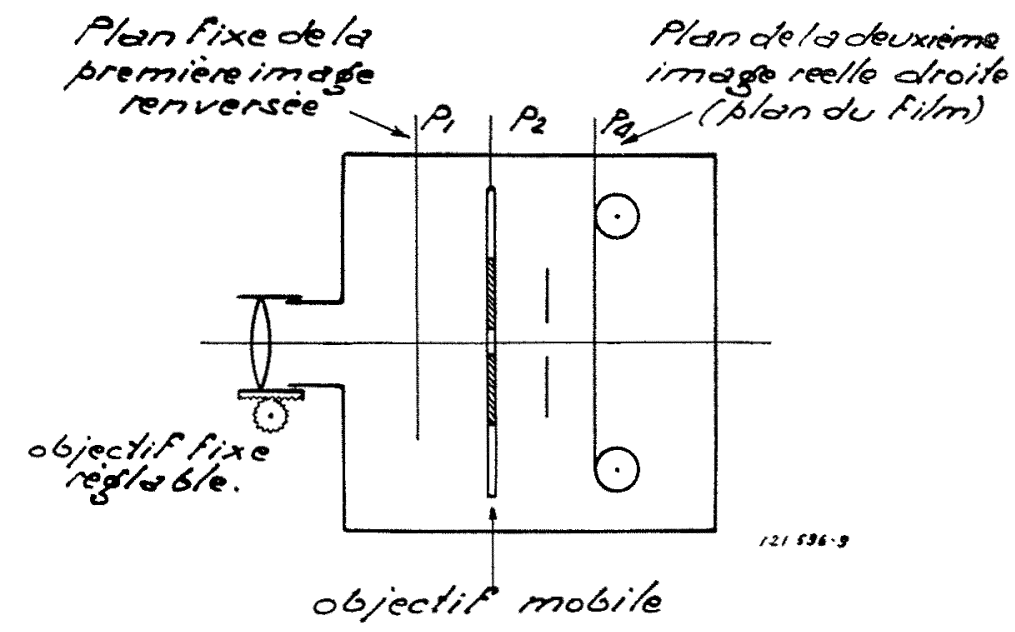

Fig. 11

une premic̀re image dans un plan bien déterminé $\mathrm{P}_{1}$ (voir fig. 11). Ciette première image sert à son tour d'objet virtuel pour le système d'objectifs mobiles qui en donnent alors une image réelle sur le film.

La distance de l'objet virtuel au plan des objectifs mobiles étant constante, la vitesse de l'image finale sera constante, et il suffira de donner au film cette vitesse pour réaliser la fixité des images par rapport au film.

$2^{\circ}$ ) Il permet d'obtenir une vitesse de déroulement inférieure à la vitesse tangentielle des objectifs.

Si nous nous arrangeons pour que le plan $\mathrm{P}_{1}$, dans laquel se forment les images virtuelles, soit en arrière du plan des objectif's mobiles $\mathrm{P}_{2}$, le film se trouvant alor's entre cres deux plans, la vitesse des images est inférieure à celle des objectifs mobiles. Nous allons le démontrer dans l'expusé qui suit.
C) Exposé du Calcul (fig. $12 a$ ).

Désignons comme précédemment par $\mathrm{P}_{1}$ le plan dans lequel doivent se former les images primaires données par l'objectif M. Ce plan est bien déterminé et fixe.

Désignons par $\mathrm{P}_{2}$ le plan contenant le centre optique des objectifs mobiles $\mathrm{O}$.

Cies objectifs mobiles ont tous, par définition, même distance focale $f$.

Soit $P_{3}$ le plan focal de ces objectifs.

$\mathrm{P}_{1}, \mathrm{P}_{2}, \mathrm{P}_{3}$ sont des plans bien déterminés et situés à des distances bien définies les unes des autres.
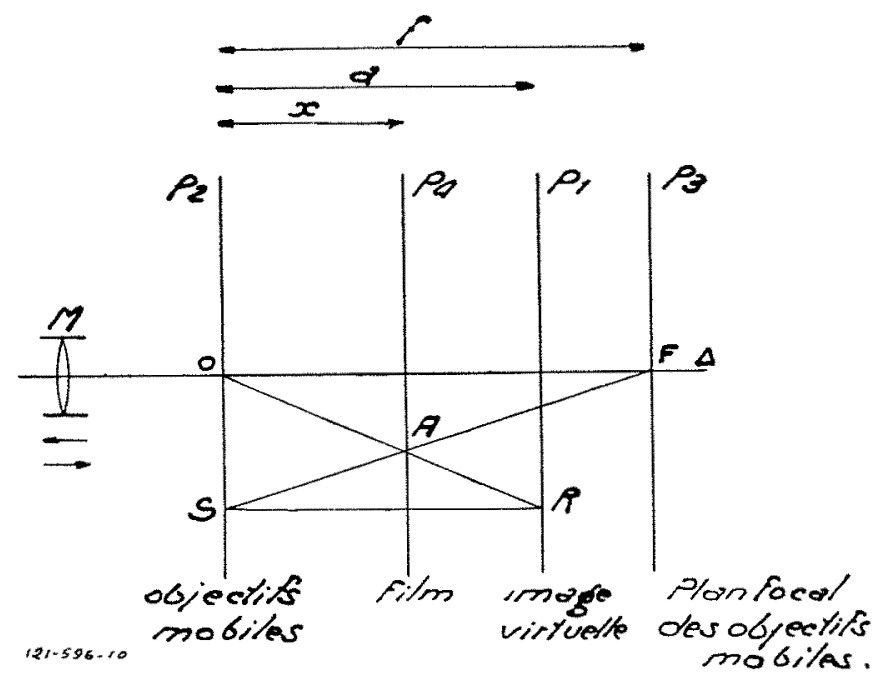

Fig. 12 a

Par définition la distance $\mathrm{P}_{2}-\mathrm{P}_{3}$ est égale à la distance focale $f$, et soit $a$ la distance de $\mathrm{P}_{2}$ à $\mathrm{P}_{1}$.

Le plan du film que nous appellerons $\mathrm{P}^{\prime \prime}$ se trouve, ainsi que nous l'avons dit, entre $P_{2}$ et $P_{1}$; nous désignerons par $x$ sa distance au plan $\mathrm{P}_{2}$, distance que nous déterminerons plus loin.

Nous supposerons, comme précédemment, que l'objectif subit un déplacement rectiligne, ce déplacement ayant lieu dans le plan de figure perpendiculairement à l'axe optique.

Ceci posé, déplaçons l'objectif $\mathrm{M}$ le long de son axe optique, de façon qu'il donne d'un point lumineux quelconque une image dans le plan $\mathrm{P}_{1}$ (condition à priori) et soit $\mathrm{R}$ ce point image.

Supposons qu'à un instant $t_{0}$ l'axe optique de l'objectif mobile qui se trouve en regard de l'ouverture soit confondu avec la droite $\Delta$.

$\left.1^{\circ}\right)$ Position de l'image réelle (fig. 12 a). - Le schéma classique de construction nous donne la position de l'image réelle A formée par l'objectif $O$ en partant de l'image virtuelle $\mathrm{R}$.

La formule des plans conjugués nous donne :

$$
\begin{aligned}
& a x=a f-f x \\
& x(a+f)=a f
\end{aligned}
$$

et

$$
x=\frac{a f}{a+f}
$$

$a$ et $f$ étant constants on en conclut que $x$ l'est aussi. donc :

Le plan dans lequel se forme l'image réelle finale, où doit se dérouler le film, esl un plan fixe, bien déterminé, situé à une distance $x=\frac{a f}{a+f} f^{d u}$ plan des centres optiques des objectifs mobiles et entièrement indépendant de leur position. 
$\left.2^{\circ}\right)$ Rapport de la vitesse de déroulemienl du film à la vitesse tangentielle des lentilles (fig. $12 b$ ).

L'objectif $O$ se déplaçant à une vitesse constante dans le sens de la flèche $f$, par exemple à l'insiant $t^{\prime}$, son axe optique occupera la position $\Delta^{\prime}$.

Le point $\mathrm{R}$ image virtuelle donnée par l'objectif $M$ n'a pas bougé, l'objectif $M$ et l'objet étant fixes par définition ;

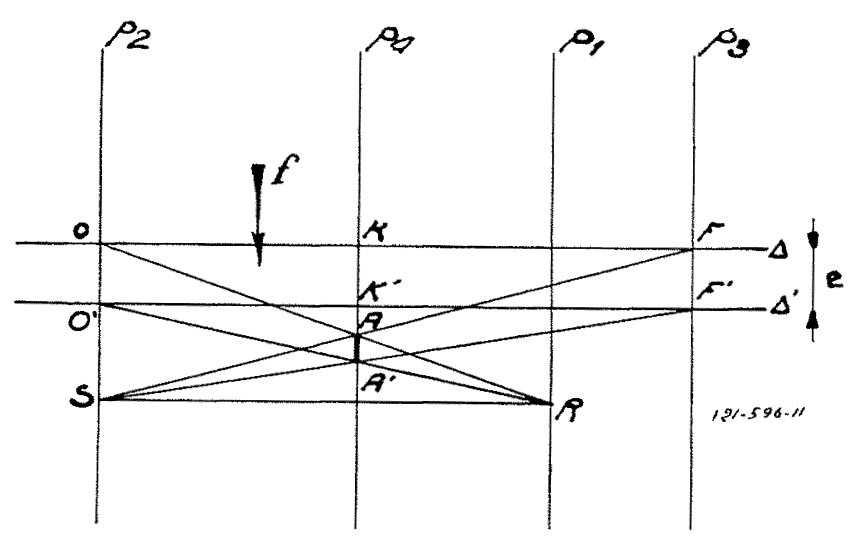

Fig. 12 b

mais les points $O, F, K$ seront venus en $O^{\prime}, F^{\prime} ; K^{\prime}, \Delta$ étant venu en $\Delta^{\prime}$.

En appclant $y^{\prime}$ le déplacemenl $\mathrm{AA}^{\prime}$ et $e$ le déplacement $\Delta \Delta^{\prime}$ Le triangle $\mathrm{S} \mathrm{F} \mathrm{F}^{\prime}$ donne $\frac{y}{e}=\frac{\mathrm{S} \mathrm{A}}{\mathrm{S} \mathrm{F}}$; mais $\frac{\mathrm{S} \mathrm{A}}{\mathrm{S} \mathrm{F}}=\frac{\mathrm{OK}}{\mathrm{OF}}=\frac{x}{f}$ d'où $y=\frac{e x}{f}$ et en remplaçant $x$ par sa valeur (1) $y=e \frac{a}{a+f}$

Pendant le temps $t-t^{\prime}$ l'objectif s'est déplacé de la dis- tance $e$, limage s'est déplacée dans le mìme sens de la distance $y=e \cdot \frac{a}{a+f}$

Si l'on désigne par

- Vi la vitesse de l'image.

- Vm la vitesse de l'objectif.

Le rapport des vitesses sen : $\frac{V i}{\mathrm{~V} m}=\frac{a}{a+f}=\mathrm{K}$

La vitesse de l'image sera tonjours injérieure à la vitesse de l'objeclif et par le choix judicieux de a el $f$ on pourra lui donner telle valeur que l'on voudra.

En particulier il n'y a plus aucune difficulté à utiliser à la fois le plus petit film du commerce (film de $8 \mathrm{~m} / \mathrm{m}$ ) el des objeclifs mobiles de dimensions convenables (diamètre supérieur à $8 \mathrm{~m} / \mathrm{m}$ ).

De plus, quelle que soit la distance de l'objet à cinémalographier, en modifiant simplement le tirage de l'objectif fixe $\mathrm{M}$, on pourna toujour's amener l'image primaire à se former dans le Plan $\mathrm{P}^{\prime \prime}$ ou, ce qui revient au même, amener l'image définitive à se former dans le plan $\mathrm{P}$, c'est-à-dire sur le film.

Le problème que nous nous étions posé est donc complètement résolu, et c'est en nous inspirant de ces considénations que nous avons construit 'un appareil cinématographique permettant de prendre 6.000 vues par seconde sur un film de $8 \mathrm{~m} / \mathrm{m}$, appareil dont nous allons donner sommairement la description.

A noter que nous avons depuis, construit d'autres appareils, et étudié, en outre, à la demande de certains laboratoires, un appareil permettant l'utilisation du film de $16 \mathrm{~m} / \mathrm{m}$.

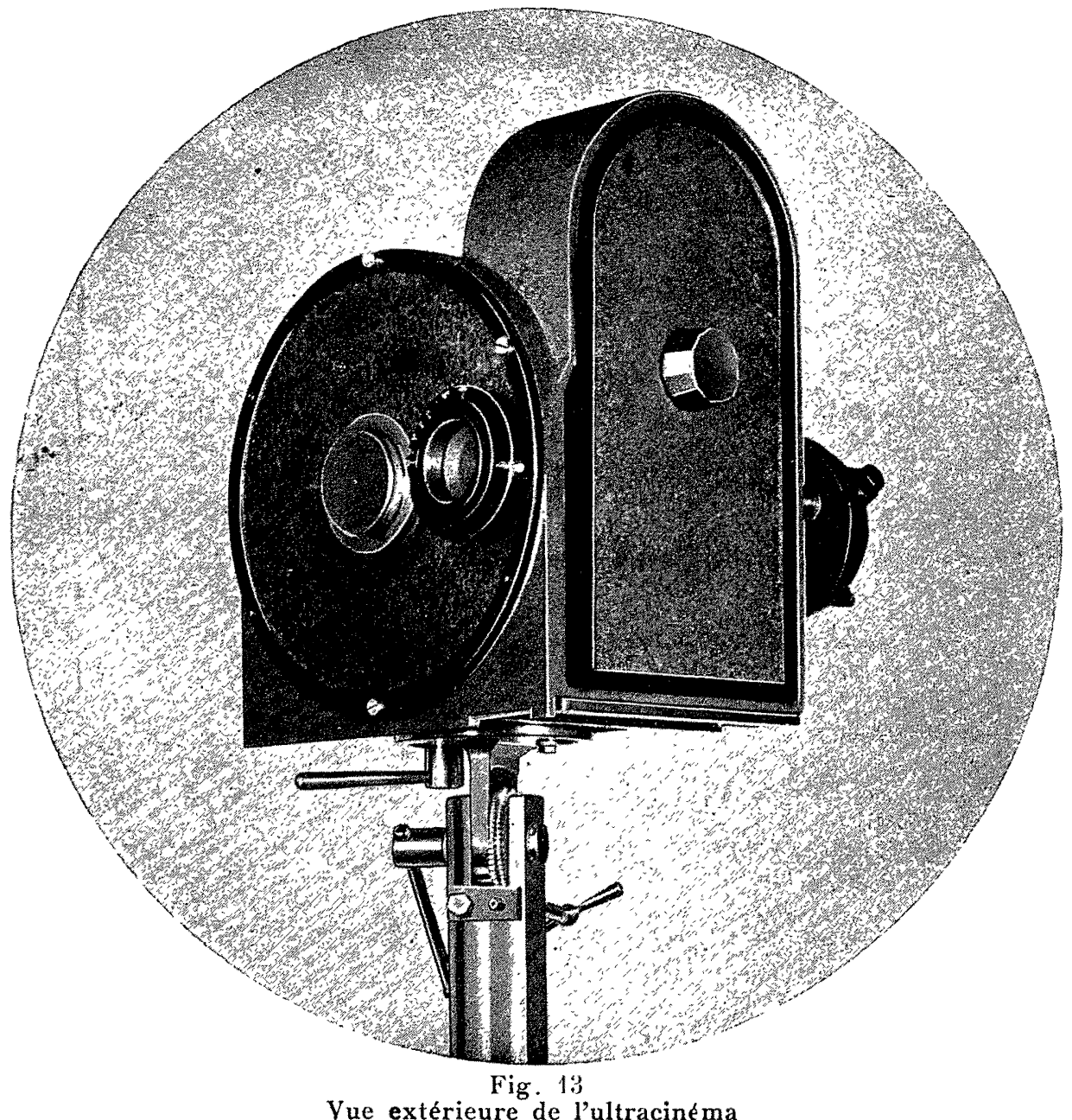

\section{Description de l'appareil cinématographique basé sur ce principe et permettant de réaliser 6.000 vues par seconde sur un film de $8 \mathrm{~m} / \mathrm{m}$}

Cet appareil (fig. 13) se compose :

- d'un dispositif optique analogue à celui décrit ci-dessus, et permettant, ainsi qu'on vient de le démontrer, de réaliser la fixité des images par rapport au film, pendant le temps de leur impression (fig. 14).

- d'un dispositif mécanique permettant de dérouller le film de façon continue, la ivitesse do déroulement du film étant à chaque instant liée à la vitesse tangentielle des objectifs mobiles par la valeur $\frac{\mathrm{V} i}{\mathrm{~V} m}=\frac{a}{a+f} \quad$ (lig. 15).

- d'un dispositif de synchronisation permettant de commander l'enraînement et l'arrêt du système moteur et la production du phénomène à étudier, le tout à des intervalles de temps déterminés (fig. 16).

- d'un moteur qui entraine les différents mécanismes (fig. 17).

Le moleur $\mathrm{H}$ entraîne en bout d'arbre un disque A portant à sa périphérie 60 objectifs de $20 \mathrm{~m} / \mathrm{m}$ de distance focale et situés à $9,5 \mathrm{~m} / \mathrm{m}$ les uns des alutres. 
Ces objectifs défilent tour à tour en face d'une ouverture derrière laquelle se déroule le film E. En avant, et sur l'axe de celte ouverlure, se trouve un objectif fixe $M$ de $25 \mathrm{~m} / \mathrm{m}$ de distance focale, dont le tirage est réglable au moyen d'une monture hélicoïdale. Le film est enroulé autour d'une

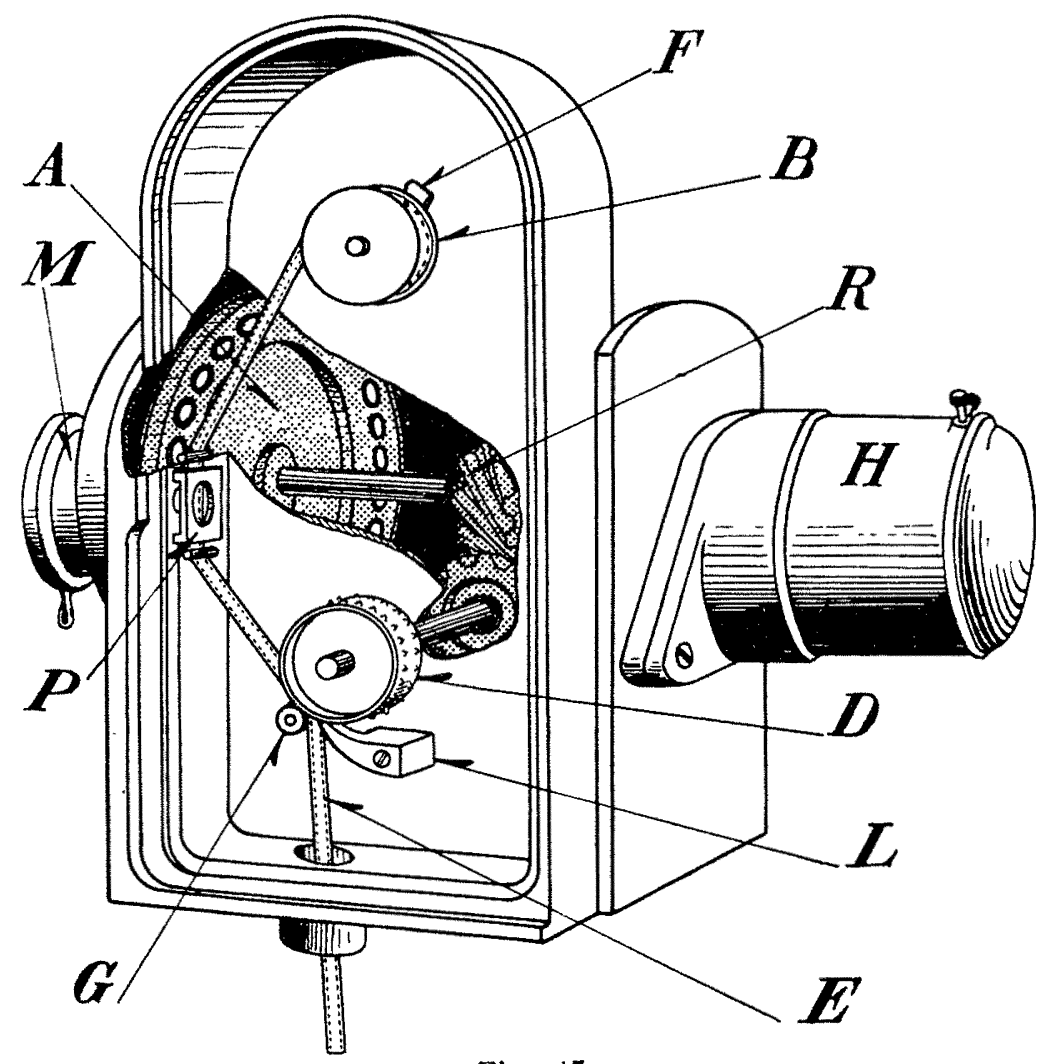

Fig. 17

bobine $\mathrm{B}$ folle sur son axe, un léger frotteur $\mathrm{F}$ l'empêche seulement de s'emballer a'u démarrage. Le film passe ensuite cntre deux platires P. La platine avant présente l'ouverture que nous avons mentionnée plus haut et une légère rainure pour le guidage du film.

La platine arrière présente également une ouverture dans laquelle s'encastre une lampe de verre. On peut ainsi examiner, au moyen d'un microscope, les images qui se forment sur le film et les amener à la netteté voulue en réglant la lentille M. Un dérouleur D, sorte de roue dentée, dont les dents s'engrènent dans les perforations du film, communique à ce dernier la vitesse uniforme désirée.

Une roulette $\mathrm{G}$ maintient le film plaqué contre le dérouleur à l'endroit où se fait l'engrênement.

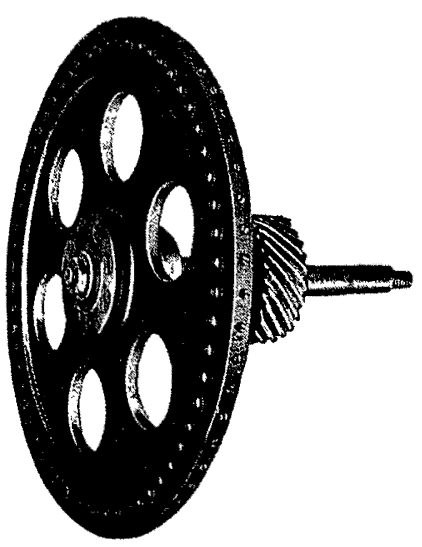

Fig. 14

Disque équipé avec ses 60 objectifs mobiles

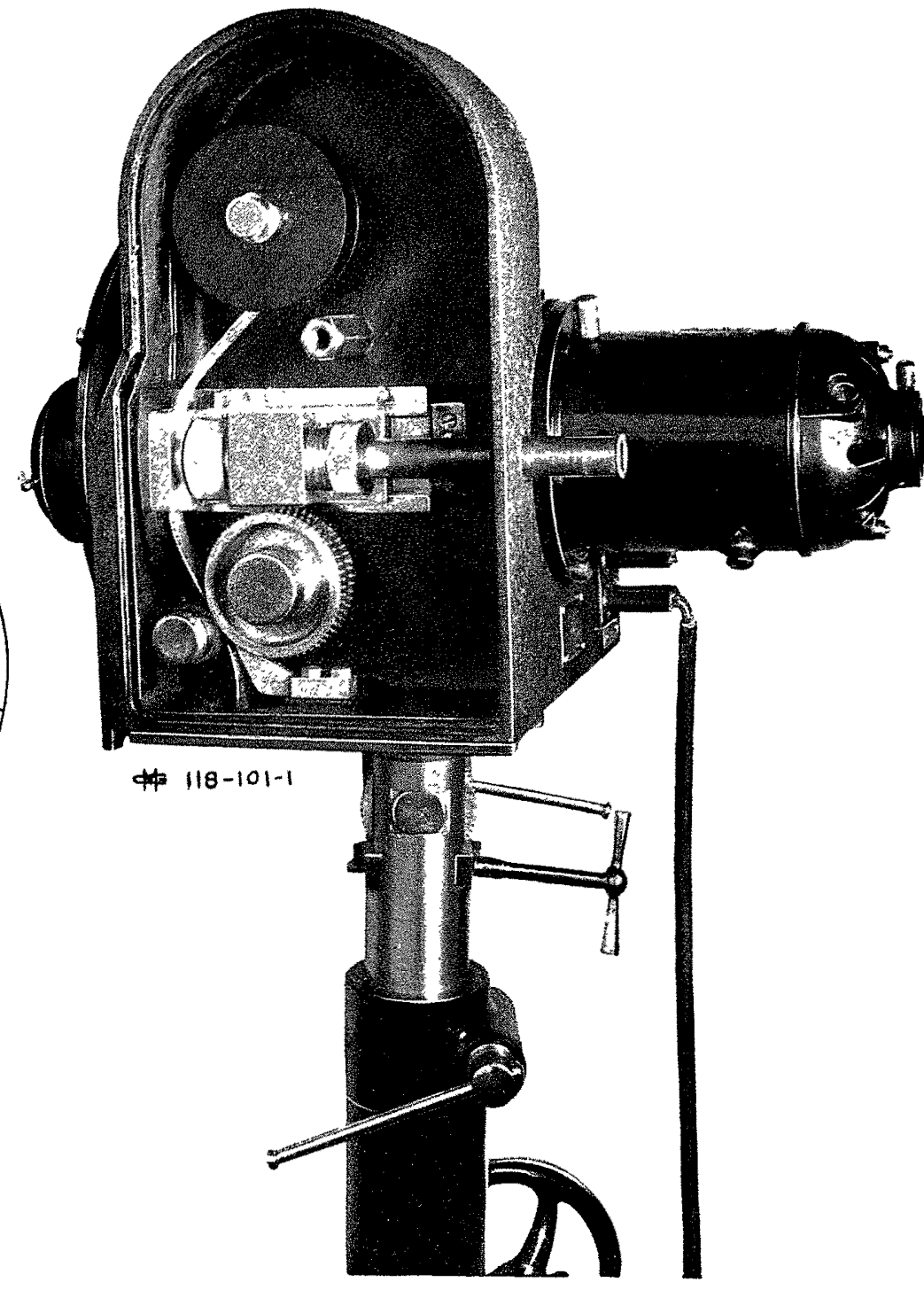

Fig. 15

Vue de l'appareil boitier ouvert montrant le mécanisme d'entrainement du film

Un doigt $L$ décolle le film à sa sortie du dérouleur et l'empêche de s'enrouler autour de ce dernier.

Finalement le film tombe en vrac dans un sac, car à la vitesse de $24 \mathrm{~m}$. par seconde, il ne peut ètre question de l'enrouler.

Un système de deux pignons hélicoïdaux $\mathrm{R}$, à angle droit, montés respectivement sur l'arbre du disque et sur l'arbre du dérouleur permet l'entraînement de ce dernier.

Tout cet ensemble nécessite un usinage de très grande précision et les différentes pièces énumérées n'ont pas d'erreurs de construction supérieures à $2 / 100$ de $\mathrm{m} / \mathrm{m}$.

Les objectifs mobiles de $20 \mathrm{~m} / \mathrm{m}$ de distance focale ne s'écartent pas de celte valeur de plus de $2,5 / 10$ de $\mathrm{m} / \mathrm{m}$. Le régllage du système étant fait pour la distance focale $\mathrm{f}$, le calcul montre que dans le cas din objectif de distance focale réelle f' la vitesse des images est légèrement différente de la vitesse du film, le rapport de ces vitesses étant :

$\mathbf{K}_{1}=-a+V \frac{\overline{a^{2}+4(a+f) f^{\prime}}}{2 f^{\prime}}$

ce qui donne dans le cas qui nous intéresse pour $\mathrm{K}_{1}$ la valeur

$$
\mathbf{K}_{1}=1,01
$$

l'image ne sera plus parfaitement immobile mais glissera légèrement d'une distance égale à $1 / 100$ de sa hauteur, glissement très acceptable en pratique.

Le moteur est un moteur shunt. Sa vitesse peut atteindre 


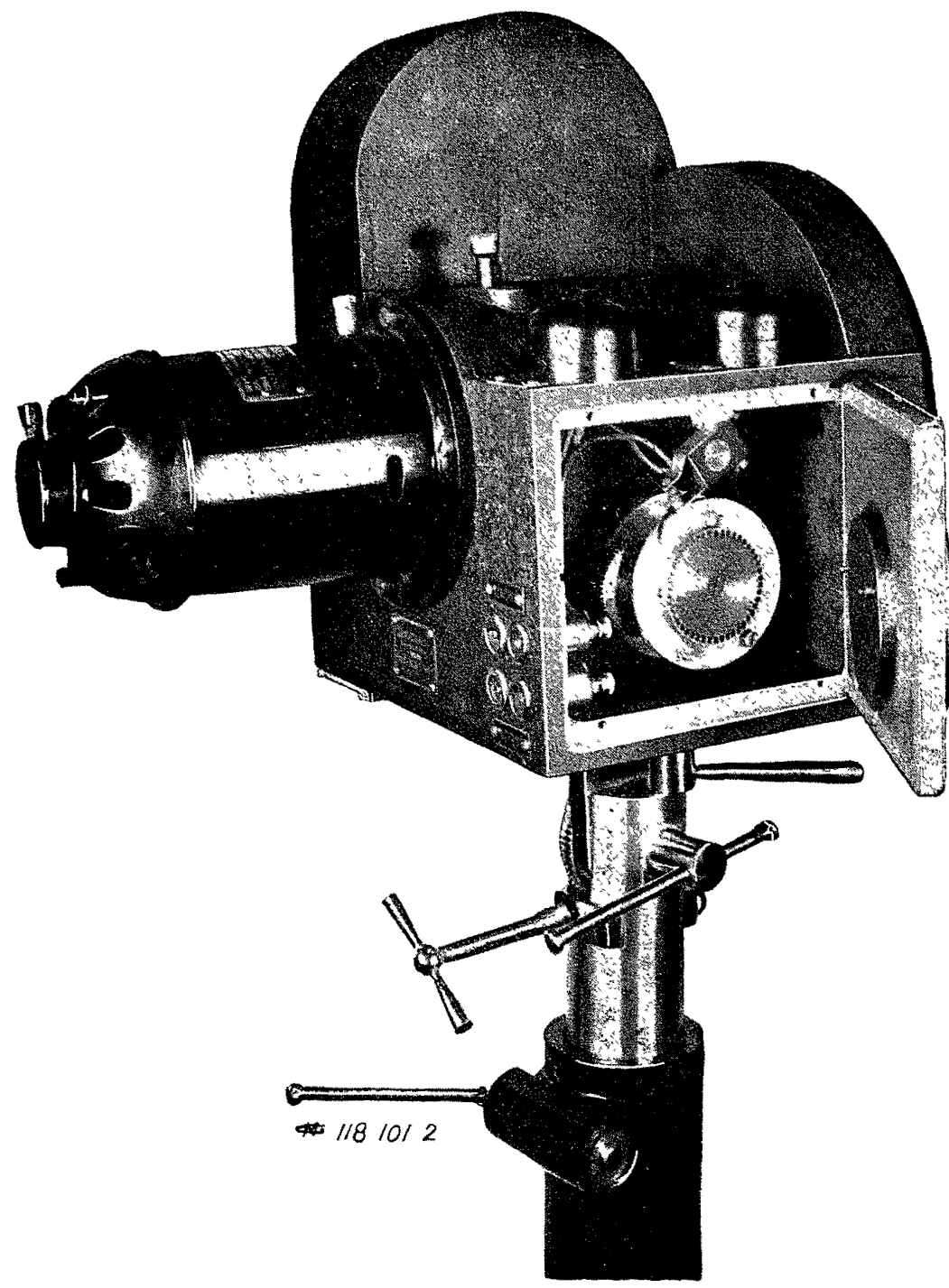

Fig. 16

Vue de l'appareil montrant le mécanisme de synchronisation entre le mouvement du film et déclenchement du phénomème.

$6.000 \mathrm{t} / \mathrm{m}$., elle est réglable en faisant varier la tension aux bornes.

A la vitesse de $6.000 \mathrm{t} / \mathrm{m}$., on obtient 6.000 images par seconde.

La vitesse de déroulement du film est alors :

$$
6.000 \times 0,038=22,8 \mathrm{~m} / \mathrm{sec} \text {. }
$$

Notons en passant que si l'on se place seulement sous l'angle de la sensibilité du film, il nous paraît difficile de dépasser beaucoup cette vitesse si l'on veut obtenir des images correcles sans une trop grande complication d'éclairage.

\section{Quelques résultats obtenus}

Afin d'illustrer cet exposé un peu aride, et pour montrer les possibilités de cet appareil, le lecteur trouvera ci-inclus trois planches illustrées.

La première planche est un agrandissement de quatre fragments de film (fig. 18).

- Ces fragments, qui représentent une ou plusieurs pha- ses de divers phénomènes d'ordres électrique, physique ou mécanique, montrent la netteté obtenue pour des enregistrements pris à différentes vitesses, ainsi que les possibilités étendues de l'appareil.

La planche II représente quelques vues sélectionnées, sur la tolalité du film ayant trait à la coupure de l'arc alternatif. (l.es numéros situés au-dessus de chaque image représentent le templs en millièmes de seconde, la première image étant prise pour origine.) (Fig. 19.)

Cet arc a été tirć entre deux électrodes métalliques s'écarlanl progressivement l'une de l'autre. L'alternance du courant est rendue très visible par l'émission de vapeurs incandescenles, au niveau de l'électrode négative. La polarité des électrodes s'inversant tous les $1 / 100$ de seconde, les électrodes paraissent ètre de véritables lances-flammes se bombarbant réciproruement à chaque 1/2 période. On remarquera, vers la fin du phénomène, plusieurs tentatives de réamorçage, puis de réels réamorçages, l'arc ayant tendance à suivre un chemin plus direct que celui emprunté par les vapeurs métalliques incandescentes, qui dessinent alors dans l'espace de fantaisies arabesques.

La planche III représente quelques vues sélectionnées sur la totalité du film se rapportant à la chute de la goutte d'eau. (fig. 20.)

On remarquera la forme parfaitement sphérique de cette goutte avant contact avec la nappe d'eau, sa pénétration sans déformation dans le sein du liquide, puis son rebondissement et le curieux appendice liquide qui semble la supportre lors de son mouvement ascentionnel, sa séparation d'avec la colonne liquide, la chute de la gouttelette et de la colonne liquide et enfin le repos du liquide.

Il est intéressant de faire remarquer à ce propos que ce dernier film a été tiré sans l'aide d'aucune source de lumière artificielle, ce qui permet de mettre en évidence de façon saisissante la gnande luminosité du dispositif optique.

Les essais effectués avec cet appareil, montrent en effet, que si l'on cinématographie un sujet déterminé, à raison de 1.000 images par seconde par exemple, les résultats photographiques obtenus sont identiques à ceux que donnerait une photographie prise au $1 / 1.000$ de seconde avec un appareil photographique ouvert à $f / 3,5$ (la sensibilité des émulsions étant évidemment la même dans les deux cas).

\section{Conclusions}

Nous croyons avoir suffisamment démontré dans ce qui précède, que par des moyens relativement simples sortant des procédés de laboratoire, il était maintenant possible d'effectuer à peu de frais, de profitables incursions dans le domaine de l'extrêmement court, par la reconstitution visuelle au ralenti de toute une catégorie d'intéressants phénomènes dont l'étude était jusqu'ici inaccessible à la plupart des chercheurs.

Notre but sera pleinement atteint si notre appareil concourt, même dans une modeste mesure, à ouvrir à ces derniers de nouvelles voies dans la recherche scientifingue de la vérité. 
PL. I

REPRODUCTION DE QUELQUES FRAGMENTS DE FILM

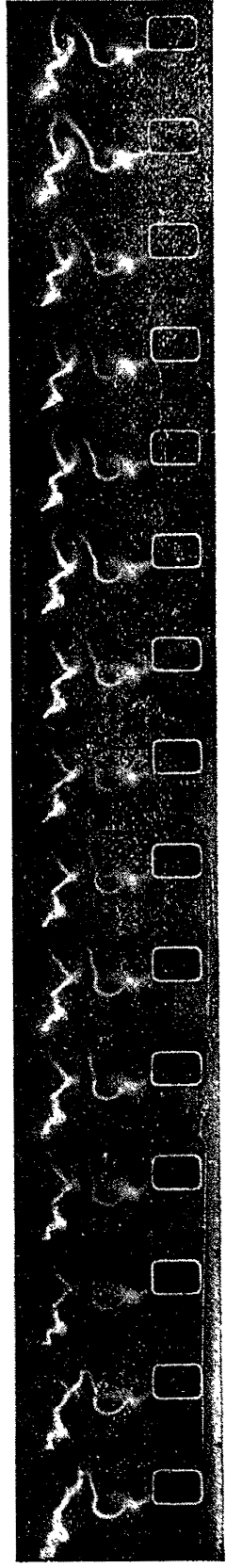

Une phase de la coupure d'un arc alternatif (3.500 images par seconde).
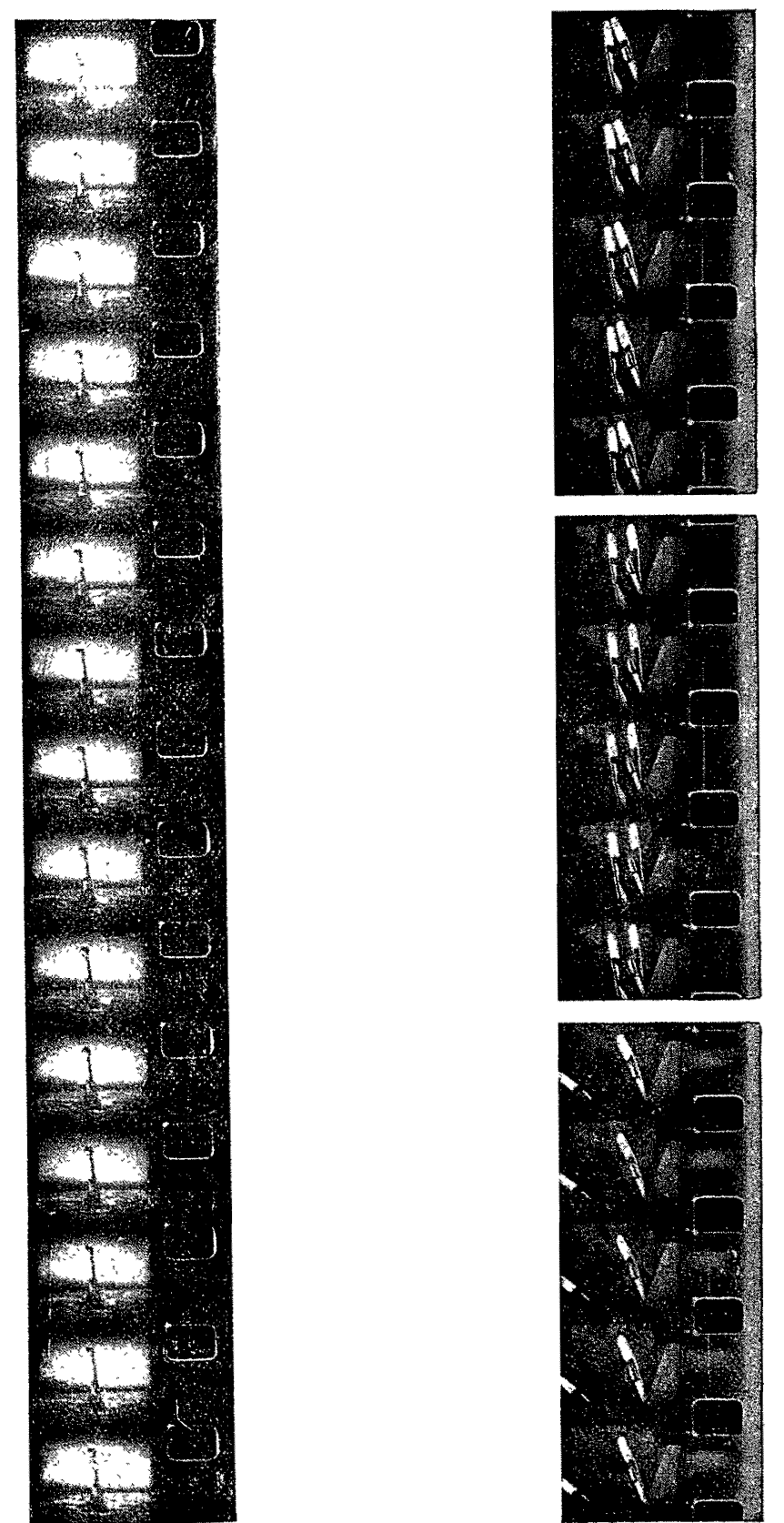

Une phase de l'ouverture des contacts d'un disjoncteur dans l'air, type

$$
\text { "Croiseurs". }
$$

(1.000 images parseconde)

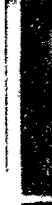
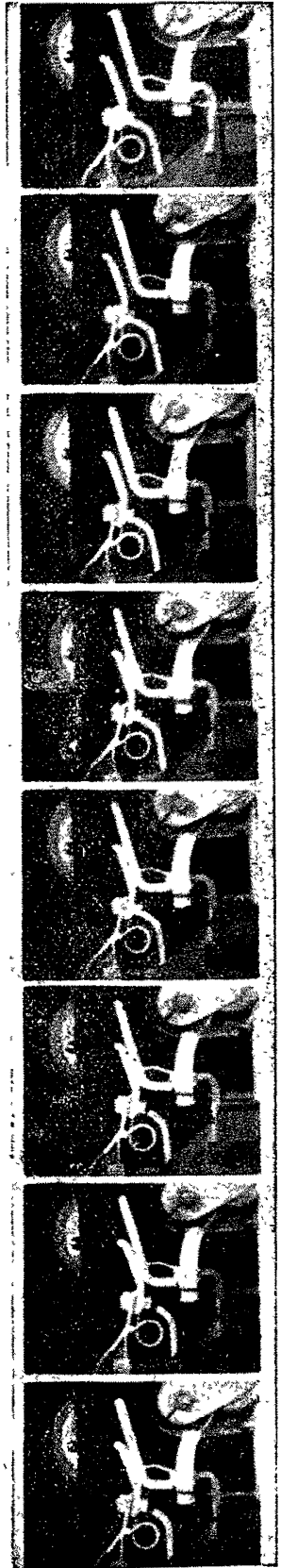

Une phase de la chute d'une goutte d'eau. 500 images
seconde).

Rebondissement de la goutte après le choc.
Etude cinématique du mouvement de roulement du contact fixe sur le contact mobile, à l'ouverture d'un contacteur. (500 images par seconde)

Les flèches indiquent le sens de la succession des images dans le temps.

Fig. 18 
Pl. II. - Cinématographie a grande vitesse (3.500 images par seconde) de la coupure d'un arg altehnatif Quelques vues sélectionnées montrant. diverses phases du phénomène

1
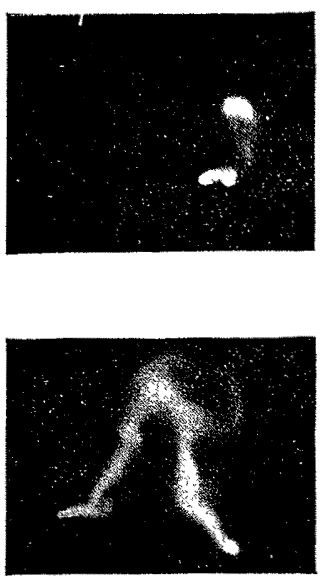

150
30
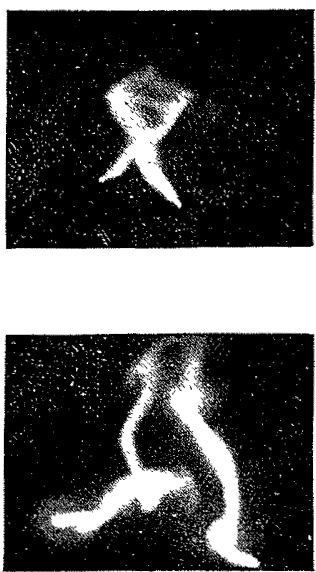

180
60
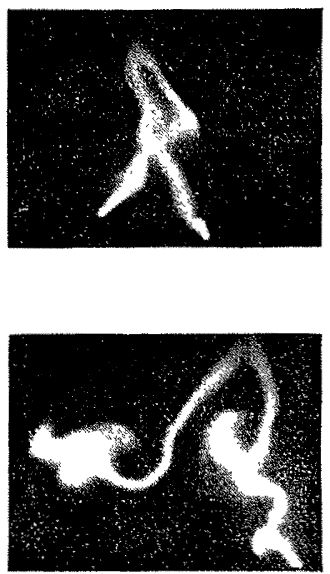

210
90
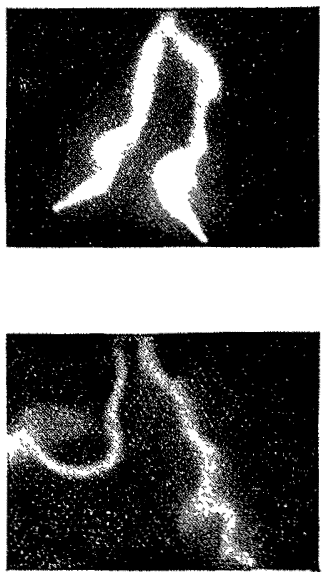

240
120
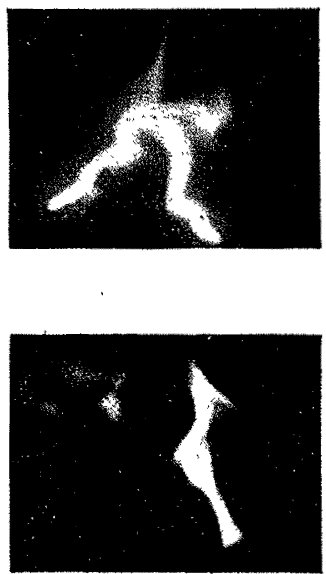

270

Durée du phénomène : $3 / 10$ de seconde. - Nombre d'images : 1.050

Fig. 19

Pl. III. - Cinématographie a moyenne vitesse (1.500 images par seconde) de la chute d'une goutte d'eau SUR UNE NAPPE D'EAU AU REPOS

Quelques vues sélectionnées montrant diverses phases du phénomène

1
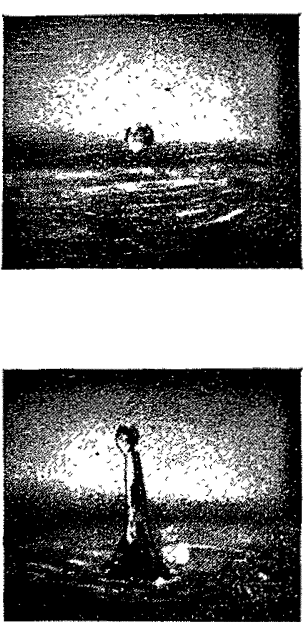

210
40
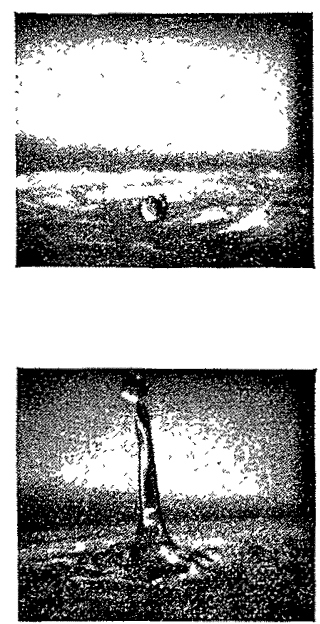

240
80
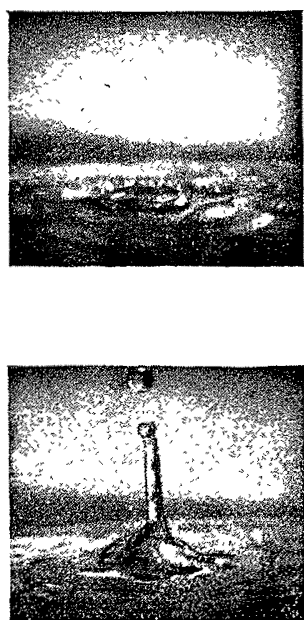

300
130
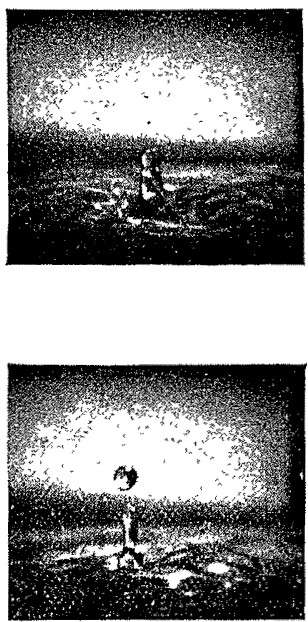

380
180
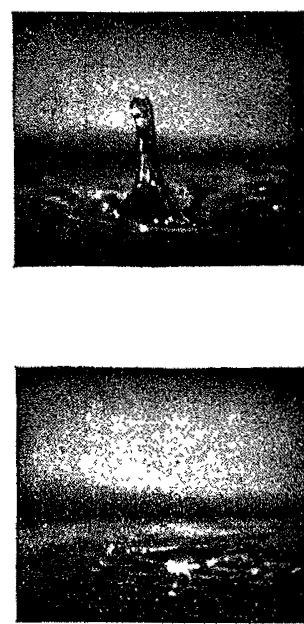

500

Durée du phénomène : 1/2 seconde. - Nombre d'images : 750

Fig. 20 